Faculty of Mathematical Sciences

\section{University of Twente}

University for Technical and Social Sciences
P.O. Box 217

7500 AE Enschede

The Netherlands

Phone: +31-53-4893400

Fax: +31-53-4893114

Email: memo@math.utwente.nl

Memorandum No. 1494

Two extensions of the Shapley value

for cooperative games

T.S.H. Driessen and D. Paulusma

JULY 1999

ISSN 0169-2690 


\title{
TWO EXTENSIONS OF THE SHAPLEY VALUE FOR COOPERATIVE GAMES
}

\author{
T.S.H. DRIESSEN AND D. PAULUSMA
}

\begin{abstract}
Two extensions of the Shapley value are given. First we consider a probabilistic framework in which certain consistent allocation rules such as the Shapley value are characterized. The second generalization of the Shapley value is an extension to the structure of posets by means of a recursive form. In the latter setting, the Shapley value for quasi-concave games is shown to be a core-allocation.
\end{abstract}

\section{INTRODUCTION}

Consider the problem of allocating some overall costs among a number of agents who have undertaken a joint venture. This allocation problem may be solved in a variety of ways, but an allocation rule that prescribes somehow a solution for the allocation problem should be justifiable on the basis of generally accepted principles. A well-known solution of cooperative games is the Shapley value ( $c f$. Shapley [1953], Roth [1988]).

A cooperative game is described by a pair $(N, c)$, where $N$ is a finite set of $n \geq 2$ players and $c: 2^{N} \rightarrow \mathbb{R}$ is a cost function satisfying $c(\emptyset)=0$.

As mentioned above, a central problem in cooperative game theory is to find a 'fair' allocation of the total costs $c(N)$ to the players. A vector $x \in \mathbb{R}^{N}$ is a cost allocation if $x$ is efficient, i.e., $x(N)=c(N)$. (Throughout the paper, for any $x \in$ $\mathbb{R}^{N}$ and $S \subseteq N$, we use the shorthand notation $x(S)=\sum_{i \in S} x_{i}$.)

An allocation rule $\psi$ prescribes for each cooperative $n$-person game $(N, c)$ exactly one allocation. The Shapley value of a game $(N, c)$ is defined as

$$
\phi_{i}(N, c)=\sum_{\substack{S \subseteq N, S \ni i}} \frac{(|S|-1) !(n-|S|) !}{n !}(c(S)-c(S \backslash i)) \quad \text { for all } i \in N .
$$

In this note, we also a (partial) order on the set of players (see, e.g., Bilbao and Edelman [1996], Faigle and Kern [1992], [1997]). Denote this partial order by $P=(N, \preceq)$. Then a cooperative game is described by a pair $(P, c)$. Furthermore, we slightly generalize the model by assuming $c$ to be given for a subfamily $\mathcal{L}(P) \subseteq$ $2^{N}$ of permitted coalitions containing the grand coalition $N$.

Date: July 21, 1999.

1991 Mathematics Subject Classification. 90D12, 90D40.

Key words and phrases. cooperative game, poset, probability distribution, Shapley value. 
Besides the subfamily $\mathcal{L}(P)=2^{N}$, we consider examples of subfamilies $\mathcal{L}(P)(c f$. Faigle and Kern [1992]) such as

(1.2) $\quad \mathcal{L}(P)=\{S \subseteq N \mid$ if $j \in S$ then $i \in S$ for all $i \preceq j\}$;

(1.3) $\mathcal{L}(P)=\{S \subseteq N \mid$ if $i, j \in S$ then $k \in S$ for all $i \preceq k \preceq j\}$.

The paper is organized as follows. In Section 2, the characterization of the Shapley value by Evans [1996] is generalized. Evans [1996] has proposed a specifically chosen probabilistic framework based on a certain "uniform" probability distribution. In this framework, the Shapley value has been characterized as the unique consistent allocation, where consistency refers to a particular property described in this section. We generalize Evans' result in that the existence and uniqueness of the consistent solution is established within a similar, but less restrictive probabilistic framework. Furthermore, we consider the case in which the set of players is (partially) ordered. However, it turns out that only subfamilies $\mathcal{L}(P)$ that contain also the complement of permitted coalitions can be considered. Hence the results do not hold for subfamilies $\mathcal{L}(P)$ such as (1.2) and (1.3). Therefore, in Section 3 , we propose another generalization of the Shapley value by an extension of the recursive formula for the Shapley value introduced by Sprumont [1990]. We show that this generalized Shapley value is a core-allocation for quasi-concave games.

\section{The Unified Probabilistic Model and Consistent Allocations}

The solution approach taken here is that the solution of the game is to be determined endogenously as the expected outcome of a probabilistic reduction of the cooperative $n$-person game to various induced two-person games. For that purpose the player set $N$ is to be partitioned into two complementary coalitions $S$ and $N \backslash S$, and from each of these two coalitions a leader ("representative") has to be chosen to cope with the bilateral division problem how to divide the total costs $c(N)$, taking into account the costs $c(S)$ and $c(N \backslash S)$ of the two coalitions involved. It is supposed that any bilateral division problem is solved by applying the so-called standard solution in that the surplus $c(N)-c(S)-c(N \backslash S)$ is charged equally to both leaders of the two coalitions, in addition to their initial costs. That is, the leader of coalition $S$ is charged the amount of $c(S)+\frac{1}{2}[c(N)-c(S)-c(N \backslash S)]$ and subsequently, this leader $i$ is obliged to charge all other agents $j, j \in S \backslash\{i\}$, of his coalition the amount $x_{j}$ in accordance with the prespecified cost vector $x \in \mathbb{R}^{N}$, and the remaining costs are allocated to the leader himself.

Since the model supposes that players split randomly into two coalitions, each with a randomly chosen leader, let $p(S, N \backslash S) \geq 0$ denote the probability of the formation of the ordered partition $(S, N \backslash S)$ and $p_{i}^{S} \geq 0$ the probability that player $i$ will be leader of coalition $S$, where $S \subsetneq N, S \neq \emptyset$, and $i \in S$. In this probabilistic framework, the expected cost allocation to player $i$ in the cooperative game $(N, c)$ with reference to the cost vector $x$ is determined by the next expression: 


$$
\sum_{\substack{S \subseteq N, S \supset i}} 2 p(S, N \backslash S)\left[\left(1-p_{i}^{S}\right) x_{i}+p_{i}^{S}\left(\frac{c(N)+c(S)-c(N \backslash S)}{2}-x(S \backslash i)\right)\right] .
$$

The factor 2 arises in (2.1) because it is supposed that the ordered partitions $(S, N \backslash S)$ and $(N \backslash S, S)$ being equally likely, that is $p(S, N \backslash S)=p(N \backslash S, S)$ for all $S \subsetneq N$, $S \neq \emptyset$. Since the probabilistic model involves a probability distribution $\{p(S, N \backslash S) \mid$ $S \subsetneq N, S \neq \emptyset\}$ over the finite set of ordered partitions of the player set and various probability distributions $\left\{\left(p_{i}^{S}\right)_{i \in S}\right\}, S \subsetneq N, S \neq \emptyset$, concerning leaders within coalitions as well, we make use of the following two assumptions:

(P1) $\sum_{\substack{S \subset N, S \neq \emptyset}} p(S, N \backslash S)=1$ or equivalently, $\sum_{\substack{S \subset N, S \ni i}} 2 p(S, N \backslash S)=1$ for all $i \in N$;

(P2) $\sum_{i \in S} p_{i}^{S}=1 \quad$ for all $S \subsetneq N, S \neq \emptyset$.

Let $(N, c)$ be a cooperative $n$-person game. An allocation $x \in \mathbb{R}^{N}$ is said to be consistent (with respect to the underlying probabilistic framework) if there is no inconsistency in what each of the players will be charged, either according to $x$ or his expected outcome as given by (2.1). That is, $x$ is a consistent allocation if and only if $x(N)=c(N)$ and the following holds: for all $i \in N$

$$
\sum_{\substack{S \subset N, S \ni i}} 2 p(S, N \backslash S)\left[\left(1-p_{i}^{S}\right) x_{i}+p_{i}^{S}\left(\frac{c(N)+c(S)-c(N \backslash S)}{2}-x(S \backslash i)\right)\right]=x_{i}
$$

or equivalently (due to $(\mathbf{P 1})$ ),

$$
\sum_{\substack{S \subset N, S \ni i}} 2 p(S, N \backslash S) p_{i}^{S}\left(\frac{c(N)+c(S)-c(N \backslash S)}{2}-x(S)\right)=0 \text { for all } i \in N .
$$

Under one additional assumption on the relevant probability distributions, the next theorem states the existence and uniqueness of a consistent allocation. Further, an explicit formula for the consistent allocation is presented. The additional assumption takes into account the probability that a fixed player will be leader of coalitions containing another variable player and requires that these probabilities are the same for all variable players.

(P3) for all $i \in N \quad \sum_{\substack{S \subseteq N, S \subseteq\{i, j\}}} p(S, N \backslash S) p_{i}^{S} \quad$ is constant for all $j \in N \backslash i$.

Let $p_{i}:=\sum_{\substack{S \subset N, S \supset i}} p(S, N \backslash S) p_{i}^{S}$ represent the probability that player $i$ will be leader.

Theorem 2.1. Suppose that $\{p(S, N \backslash S) \mid S \subsetneq N, S \neq \emptyset\}$ and $\left\{\left(p_{i}^{S}\right)_{i \in S}\right\}, S \subsetneq N$, $S \neq \emptyset$, satisfy $(\mathbf{P 1}),(\mathbf{P 2})$ and $(\mathbf{P 3})$. Let $(N, c)$ be a cooperative n-person game. Then there exists a unique consistent allocation $x \in \mathbb{R}^{N}$ and it is given, for all $i \in N$, by

(2.3) $x_{i}=\left[1-(n-1) p_{i}\right] c(N)+(n-1) \sum_{\substack{S \subseteq N, S \subseteq j}} p(S, N \backslash S) p_{i}^{S}[c(S)-c(N \backslash S)]$. 
Proof: In view of assumption $(\mathbf{P 3})$ we write

$$
\bar{p}_{i}:=\sum_{\substack{S \subseteq N, S \supseteq\{i, j\}}} p(S, N \backslash S) p_{i}^{S} \quad \text { for all } i \in N \text { and all } j \in N \backslash i \text {. }
$$

Let $(N, c)$ be a cooperative game, $i \in N$ and $x \in \mathbb{R}^{N}$ a cost allocation for the game $(N, c)$. By some careful, but straightforward combinatorial computations concerning some double sum, we arrive at the following chain of equalities:

$$
\begin{aligned}
& \sum_{\substack{S \subseteq N, S \ni i}} 2 p(S, N \backslash S) p_{i}^{S} x(S) \\
= & \sum_{\substack{S \subseteq N, S \ni i}} 2 p(S, N \backslash S) p_{i}^{S} x_{i}+\sum_{\substack{S \subseteq N, S \ni i}} 2 p(S, N \backslash S) p_{i}^{S} \sum_{j \in S \backslash i} x_{j} \\
= & 2 p_{i} x_{i}+2 \sum_{j \in N \backslash i} x_{j} \sum_{\substack{S \subseteq N, S \supseteq i, j\}}} p(S, N \backslash S) p_{i}^{S} \quad \text { (by definition of } p_{i} \text { ) } \\
= & 2 p_{i} x_{i}+2 x(N \backslash i) \bar{p}_{i} \quad \text { (by definition of } \bar{p}_{i} \text {, see (2.4)) } \\
= & \left.2 p_{i} x_{i}+2\left[c(N)-x_{i}\right] \bar{p}_{i} \quad \text { (by efficiency of } x\right) \\
= & 2\left[p_{i}-\bar{p}_{i}\right] x_{i}+2 \bar{p}_{i} c(N) . \quad
\end{aligned}
$$

Hence the consistency constraint (2.2) for the allocation $x$ reduces to the following equality: for all $i \in N$

$$
\sum_{\substack{S \subseteq N, S \ni i}} p(S, N \backslash S) p_{i}^{S}[c(S)-c(N \backslash S)]+\left[p_{i}-2 \bar{p}_{i}\right] c(N)=2\left[p_{i}-\bar{p}_{i}\right] x_{i} .
$$

In order to deduce (2.3) from the latter equality, it remains to establish that $p_{i}-\bar{p}_{i}=$ $\frac{1}{2(n-1)}$ for all $i \in N$. Actually, we claim that the following results hold:

$\sum_{i \in N} p_{i}=1 ; \quad \sum_{j \in N \backslash i} \bar{p}_{j}=\frac{1}{2}-p_{i} \quad$ and $\quad p_{i}-\bar{p}_{i}=\frac{1}{2(n-1)} \quad$ for all $i \in N$.

To prove the first statement in (2.5), some straightforward combinatorial computations concerning some double sum and applying the assumptions (P2) and (P1) 
respectively, yield the following chain of equalities:

$$
\begin{aligned}
\sum_{i \in N} p_{i} & =\sum_{i \in N} \sum_{\substack{S \subseteq N, S \ni i}} p(S, N \backslash S) p_{i}^{S} \quad \text { (reverse the order of the double sum) } \\
& =\sum_{\substack{S \subseteq N, S \neq \emptyset}} p(S, N \backslash S) \sum_{i \in S} p_{i}^{S} \\
& \stackrel{(P 2)}{=} \quad \sum_{\substack{S \subseteq N, S \neq \emptyset}} p(S, N \backslash S) \cdot 1 \\
& \stackrel{(P 1)}{=} 1 .
\end{aligned}
$$

To prove the second statement in (2.5), a similar reasoning, for all $i \in N$, yields the following chain of equalities:

$$
\begin{aligned}
\sum_{j \in N \backslash i} \bar{p}_{j} & \stackrel{\text { (2.4) }}{=} \sum_{j \in N \backslash i} \sum_{\substack{S \subseteq N, S \supseteq i i, j\}}} p(S, N \backslash S) p_{j}^{S} \quad \text { (reverse the order of the double sum) } \\
& =\sum_{\substack{S \subseteq N, S \ni i}} p(S, N \backslash S) \sum_{j \in S \backslash i} p_{j}^{S} \\
& \stackrel{(P 2)}{=} \sum_{\substack{S \subseteq N, S \ni i}} p(S, N \backslash S)\left[1-p_{i}^{S}\right] \\
& \stackrel{(P 1)}{=} \quad \frac{1}{2}-p_{i} \quad \text { (by definition of } p_{i} \text { ). }
\end{aligned}
$$

From the obtained equalities $\sum_{j \in N} p_{j}=1$ and $\sum_{j \in N} \bar{p}_{j}=\frac{1}{2}-p_{i}+\bar{p}_{i}$, we deduce that $\sum_{j \in N}\left[p_{j}-\bar{p}_{j}\right]=\frac{1}{2}+p_{i}-\bar{p}_{i}$ for all $i \in N$. It follows immediately that $p_{i}-\bar{p}_{i}=\frac{1}{2(n-1)}$ for all $i \in N$. That is, (2.5) holds which completes the full proof of the theorem.

The following corollary presents the result by Evans [1996]. The straightforward proof is left to the reader.

Corollary 2.1. Let the "uniform" probability distribution $\{p(S, N \backslash S) \mid S \subsetneq N, S \neq$ $\emptyset\}$ be given by $p(S, N \backslash S):=\left[(n-1) \cdot\left(\begin{array}{c}n \\ |S|\end{array}\right)\right]^{-1}$ for all $S \subsetneq N, S \neq \emptyset$, and moreover, let $p_{i}^{S}:=\frac{1}{|S|}$ for all $S \subsetneq N, S \neq \emptyset$, and $i \in S$. Then (P1), (P2) and (P3) hold and the unique consistent allocation $x \in \mathbb{R}^{N}$ for a cooperative game $(N, c)$ agrees with the Shapley value, i.e., (2.3) reduces to

$$
x_{i}=\sum_{\substack{S \subseteq N, \bar{S} \Im i}} \frac{(|S|-1) !(n-|S|) !}{n !}(c(S)-c(N \backslash S)),
$$


which is equivalent to (1.1).

In the rest of this section, we suppose that $(\mathbf{P 1}),(\mathbf{P 2})$ and $(\mathbf{P 3})$ hold. Define the consistent allocation rule as the rule that assigns to every cooperative game $(N, c)$ its unique consistent allocation as given by (2.3). We say the consistent allocation rule possesses the dummy player property if for every cooperative game $(N, c)$, the consistent allocation $x \in \mathbb{R}^{N}$ satisfies $x_{i}=c(\{i\})$ for every dummy player $i$ in the game $(N, c)$. Here player $i$ is called a dummy if $c(S)-c(S \backslash i)=c(\{i\})$ for all $S \subseteq N$ with $i \in S$.

The next theorem presents two characterizations of the dummy player property to hold true for the consistent allocation rule. A first characterization refers to necessary and sufficient conditions on the underlying probability distributions stating that the probability of a player's leadership within a nontrivial coalition (with reference to the corresponding ordered partition of the player set) is the same for both the coalition and its complementary coalition enlarged with the given player. The second characterization requires the consistent allocation rule to be a probabilistic marginalistic allocation rule ( $c f$. Weber [1988]) in that the allocation to any player is some expected outcome of his marginal contributions in the game. Note that the Shapley value is an example of a consistent allocation rule that satisfies the dummy player property.

Proposition 2.1. For any coalition $S \subseteq N$ and any player $i \in N$ with $i \in S$, define the "enlarged" complementary coalition $S^{c+i}$ to be $(N \backslash S) \cup\{i\}$. Then the following three statements are equivalent.

(D1) The consistent allocation rule possesses the dummy player property.

(D2) The underlying probability distributions satisfy the next conditions:

$$
\begin{aligned}
p(S, N \backslash S) p_{i}^{S} & =p\left(S^{c+i}, N \backslash S^{c+i}\right) p_{i}^{S^{c+i}} \quad \text { whenever }\{i\} \subsetneq S \subsetneq N ; \\
p(\{i\}, N \backslash i) \cdot p_{i}^{\{i\}} & =\frac{1}{n-1}-p_{i} \quad \text { for all } i \in N .
\end{aligned}
$$

(D3) The consistent allocation rule is a probabilistic marginalistic allocation rule, that is, for every $i \in N$, there exists a collection of non-negative real numbers $\left\{q_{i}^{S} \mid S \subseteq N, S \ni i\right\}$ satisfying $\sum_{\substack{S \subseteq N, S \ni i}} q_{i}^{S}=1$ such that, for every cooperative game $(N, c)$, the consistent allocation $x_{i}$ to any player $i$ is of the following form:

$$
x_{i}=\sum_{\substack{S \subseteq N, S \ni i}} q_{i}^{S}[c(S)-c(S \backslash i)] .
$$

Proof:

(a) (D1) implies (D2).

Suppose the consistent allocation rule possesses the dummy player property. Let $S \subseteq N$ and $i \in N$ with $i \in S, S \neq\{i\}$. Define the cooperative game $(N, \bar{c})$ by $\bar{c}(S):=1, \bar{c}(S \backslash i):=1$ and $\bar{c}(T):=0$ otherwise. Clearly, player $i$ is a dummy in the game $(N, \bar{c})$. Denote the consistent allocation of the game $(N, \bar{c})$ by $x \in \mathbb{R}^{N}$ 
as given by (2.3). The dummy player property of the consistent allocation rule yields $x_{i}=\bar{c}(\{i\})=0$. However, by (2.3), $x_{i}$ can be determined as follows by distinguishing two cases:

$$
\begin{array}{ll}
x_{i}=1-(n-1) p_{i}-(n-1) p(\{i\}, N \backslash i) p_{i}^{\{i\}} & \text { if } S=N \\
x_{i}=(n-1)\left[p(S, N \backslash S) p_{i}^{S}-p\left(S^{c+i}, N \backslash S^{c+i}\right) p_{i}^{S^{c+i}}\right] & \text { if } S \neq N .
\end{array}
$$

From this, together with $x_{i}=0$, we conclude that (D1) implies (D2).

(b) (D2) implies (D3).

Suppose (D2) holds. Then for all $i \in N$ the following chain of equalities holds.

$$
\begin{aligned}
& \sum_{\substack{S \subset N,\langle i \backslash \leq S}} p(S, N \backslash S) p_{i}^{S}[c(S)-c(N \backslash S)] \\
& \stackrel{(D 2)}{=} \quad \frac{1}{2} \sum_{\substack{S \subset N,\langle i \backslash \subseteq}} p(S, N \backslash S) p_{i}^{S}\left[c(S)-c(N \backslash S)+c\left(S^{c+i}\right)-c\left(N \backslash S^{c+i}\right)\right] \\
& =\quad \frac{1}{2} \sum_{\substack{S \subset N,\langle i\rangle \subseteq S}} p(S, N \backslash S) p_{i}^{S}\left[c(S)-c(S \backslash i)+c\left(S^{c+i}\right)-c\left(S^{c+i} \backslash i\right)\right] \\
& \stackrel{(D 2)}{=} \sum_{\substack{S \subset N,\langle i\rangle \subseteq S}} p(S, N \backslash S) p_{i}^{S}[c(S)-c(S \backslash i)]
\end{aligned}
$$

Using this partial result, we deduce by straightforward calculations that, for all $i \in N$ the consistent allocation $x_{i}$ as given by (2.3) reduces as follows:

$$
\begin{aligned}
x_{i} & \stackrel{(2.3)}{=} \quad\left[1-(n-1) p_{i}\right] c(N)+(n-1) \sum_{\substack{S \subseteq N, S \ni i}} p(S, N \backslash S) p_{i}^{S}[c(S)-c(N \backslash S)] \\
& =\quad\left[1-(n-1) p_{i}\right] c(N)+(n-1) p(\{i\}, N \backslash i) p_{i}^{\{i\}}[c(\{i\})-c(N \backslash i)] \\
& +\quad(n-1) \sum_{\substack{S \subseteq N,\langle i\} \subseteq S}} p(S, N \backslash S) p_{i}^{S}[c(S)-c(S \backslash i)] \\
& \stackrel{(D 2)}{=} \quad\left[1-(n-1) p_{i}\right][c(N)-c(N \backslash i)] \\
& +\quad(n-1) \sum_{\substack{S \subseteq N, S \ni i}} p(S, N \backslash S) p_{i}^{S}[c(S)-c(S \backslash i)] .
\end{aligned}
$$

Hence, for all $i \in N$ the consistent allocation $x_{i}$ is of the form given by (D3) by 
choosing $q_{S}^{i}:=(n-1) p(S, N \backslash S) p_{i}^{S}$ for all $S \subsetneq N$ with $i \in S$ and $q_{N}^{i}:=1-(n-$ 1) $p_{i}$. Note that for all $i \in N$, by definition of $p_{i}$,

$$
\sum_{\substack{S \subset N, S \ni i}} q_{S}^{i}=(n-1) \sum_{\substack{S \subset N, S \ni i}} p(S, N \backslash S) p_{i}^{S}+q_{N}^{i}=(n-1) p_{i}+q_{N}^{i}=1 .
$$

This completes the proof of (b).

(c) (D3) implies (D1).

This implication is trivial by the definition of a dummy player and the assumption on the collection $\left\{q_{i}^{S} \mid S \subseteq N, S \ni i\right\}$ for any player $i \in N$.

Up to now, we have only treated the case in which the set of players was unordered. Let $\mathcal{L}(P)$ be a subfamily of permitted coalitions depending on a poset $P=(N, \preceq)$. Within our probabilistic framework, this can be modelled by making the probability $p(S, N \backslash S)$ equal to zero whenever $S$ or $N \backslash S \notin \mathcal{L}(P)$. It is clear that we have to make the following assumption:

(C) $S \in \mathcal{L}(P)$ if and only if $N \backslash S \in \mathcal{L}(P)$ for all $S \subseteq N$.

As an extra condition one could decide that only a player $i \in S$ for which there does not exist a player $j \in S$ with $j \succ i$ can be leader of $S$. This can be modelled by making the probability $p_{j}^{S}$ equal to zero for all players $j \in S$ for which there exists a player $k \in S$ with $j \prec k$. If the (adjusted) probability distributions $\{p(S, N \backslash S) \mid$ $S \subsetneq N, S \neq \emptyset\}$ and $\left\{\left(p_{i}^{S}\right)_{i \in S}\right\}, S \subsetneq N, S \neq \emptyset$ satisfy the conditions (P1), (P2) and $(\mathbf{P 3})$, the results presented in this section stay valid.

However, subfamilies $\mathcal{L}(P)$ such as (1.2) and (1.3) do not satisfy $(\mathbf{C})$. Therefore, in the next section, we propose another approach that also yields a generalized Shapley value.

\section{The Recursive Shapley Value}

In this section, the recursive formula for the Shapley value presented by Sprumont [1990] is treated. This formula has only been defined for cooperative games $(P, c)$ where $\mathcal{L}(P)=2^{N}$. We give a generalization of this formula that holds for a larger class of subfamilies $\mathcal{L}(P)$.

Consider a coalition $T \subseteq N$ and restrict the cost function $c: 2^{N} \rightarrow \mathbb{R}$ to $2^{T}$. A vector $x \in \mathbb{R}^{T}$ is called a cost allocation if $x(T)=c(T)$.

The Shapley value of a game $(T, c)$ is defined as

$$
\phi_{i}(T, c)=\sum_{\substack{S \subseteq T, S \ni i}} \frac{(|S|-1) !(|T|-|S|) !}{|T| !}(c(S)-c(S \backslash i)) \quad \text { for all } i \in T .
$$

Sprumont [1990] proved the following recursive formula for the Shapley value of a game $(T, c)$ for all non-empty coalitions $T \subseteq N$.

$$
\phi_{i}(T, c)=\frac{1}{|T|}\left(c(T)-c(T \backslash i)+\sum_{j \in T \backslash i} \phi_{i}(T \backslash j, c)\right) \quad \text { for all } i \in T .
$$


Consider a subfamily $\mathcal{L}(P)$. A player $i$ is called unrestricted in a coalition $S \in$ $\mathcal{L}(P)$ if $\mathcal{L}(P)$ contains $S \backslash i$. Denote the set of unrestricted players in $S$ by $S^{*}$, i.e., $S^{*}=\{i \in S \mid S \backslash i \in \mathcal{L}(P)\}$. We make use of the following assumption.

(A) $S^{*}$ is non-empty for all $S \in \mathcal{L}(P) \backslash \emptyset$.

Because of this assumption, a coalition $T \in \mathcal{L}(P)$ exists for every size $0 \leq|T| \leq n$.

A poset $(T, \preceq)$, where $T$ is a permitted coalition, is obtained from $P$ in the following way: $i \preceq j$ in $(T, \preceq)$ if and only if $i \preceq j$ in $P$. Let $\mathcal{L}((T, \preceq))$ be the subfamily of permitted coalitions restricted to $(T, \preceq)$, i.e., $\mathcal{L}((T, \preceq))=2^{T} \cap \mathcal{L}(P)$. An allocation rule $\psi$ prescribes for each cooperative game $((T, \preceq), c)$ exactly one allocation. We use the notations $\mathcal{L}(T)$ instead of $\mathcal{L}((T, \preceq))$ and, for an allocation rule $\psi, \psi(T, c)$ instead of $\psi((T, \preceq), c)$ if the structural context is clear.

It is easy to see that, besides $\mathcal{L}(P)=2^{N},(1.2)$ and (1.3) also satisfy (A). Note that in (1.2), $S^{*}=S^{+}$and in (1.3), $S^{*}=S^{+} \cup S^{-}$, where $S^{+}$denotes the set of maximal players and $S^{-}$denotes the set of minimal players. A player $i \in S$ is called maximal in $S$ if there does not exist a player $j \in S$ with $j \succ i$. A player $i \in S$ is called minimal in $S$ if there does not exist a player $j \in S$ with $j \prec i$.

From now on, we only consider subfamilies $\mathcal{L}(P)$ containing $N$ that satisfy (A). Consider a fixed game $(P, c)$. For all $T \in \mathcal{L}(P)$, we define the vector $\phi^{r}(T, c) \in \mathbb{R}^{T}$ as

$$
\phi_{i}^{r}(T, c)=\sum_{\substack{S \in \mathcal{L}(T), S^{*} \ni i}} \gamma(T, S)(c(S)-c(S \backslash i)) \quad \text { for all } i \in T,
$$

where for all $T \in \mathcal{L}(P), S \in \mathcal{L}(T), S \neq \emptyset$, the coefficients $\gamma(T, S)$ are recursively given by

$$
\gamma(T, S)= \begin{cases}\frac{1}{\left|T^{*}\right|} & \text { if } S=T \\ \frac{1}{\left|T^{*}\right|} \sum_{j \in T^{*} \backslash S} \gamma(T \backslash j, S) & \text { otherwise. }\end{cases}
$$

Before proving that $\phi^{r}$ is an allocation rule, we first show that $\phi^{r}$ is a generalization of the Shapley value.

Proposition 3.1. For all $T \in \mathcal{L}(P), T \neq \emptyset, \phi^{r}(T, c)=\phi(T, c)$ if $\mathcal{L}(T)=2^{T}$.

Proof: First note that $S^{*}=S$ for all $S \subseteq T$ if $\mathcal{L}(T)=2^{T}$. Hence, by (3.1), it suffices to show that

$$
\gamma(T, S)=\frac{(|T|-|S|) !(|S|-1) !}{|T| !} \text { for all } S \subseteq T, S \neq \emptyset .
$$

By assumption (A), we can use induction on $|T|$. Note that $\mathcal{L}(S)=2^{S}$ for all $S \subseteq T$ if $\mathcal{L}(T)=2^{T}$.

If $|T|=1$ then $\gamma(T, T)=1$. Suppose $|T|>1$. We have, by definition,

$$
\gamma(T, T)=\frac{1}{|T|}=\frac{(|T|-|T|) !(|T|-1) !}{|T| !} .
$$


If $S \subsetneq T, S \neq \emptyset$, we have

$$
\begin{aligned}
\gamma(T, S) & =\frac{1}{|T|} \sum_{j \in T \backslash S} \gamma(T \backslash j, S) \\
& =\sum_{j \in T \backslash S} \frac{(|T|-|S|-1) !(|S|-1) !}{(|T|-1) !|T|} \\
& =\frac{(|T|-|S|) !(|S|-1) !}{|T| !} .
\end{aligned}
$$

In the following theorem, we show that $\phi^{r}(T, c)$ is a cost allocation for all $T \in$ $\mathcal{L}(P)$. Furthermore, $\phi_{i}^{r}(T, c)$ can easily be computed for all $T \in \mathcal{L}(P), T \neq \emptyset$ if $\phi_{i}^{r}(T \backslash j, c)$ is known for all $j \in T^{*} \backslash i$. It turns out that if $i$ is an unrestricted player in the permitted coalition $T, \phi_{i}^{r}(T, c)$ is the sum of the marginal contribution $c(T)-c(T \backslash i)$ and the cost allocations to $i$ in the games $(T \backslash j, c)$, where $j \neq i$ is also an unrestricted player in $T$, divided by the number of unrestricted players in $T$. If $i \in T$ is not an unrestricted player, $\phi_{i}^{r}(T, c)$ is simply the average of the cost allocations to $i$ in the games with one unrestricted player less. Therefore, we call $\phi^{r}$ the Recursive Shapley value (see also Proposition 3.1).

Theorem 3.1. For all $T \in \mathcal{L}(P), T \neq \emptyset, \phi^{r}(T, c)$ is an allocation that can be determined recursively by

$$
\phi_{i}^{r}(T, c)= \begin{cases}\frac{1}{\left|T^{*}\right|}\left[c(T)-c(T \backslash i)+\sum_{j \in T^{*} \backslash i} \phi_{i}^{r}(T \backslash j, c)\right] & \text { for all } i \in T^{*} \\ \frac{1}{\left|T^{*}\right|} \sum_{j \in T^{*}} \phi_{i}^{r}(T \backslash j, c) & \text { for all } i \in T \backslash T^{*} .\end{cases}
$$

Proof: First we will prove that the recursive form holds. This will be done by some combinatorial computations concerning some double sum.

Suppose $T \in \mathcal{L}(P)$ and $i \in T$. There are two cases.

(a) $i \in T^{*}$. 
We have

$$
\begin{aligned}
\phi_{i}^{r}(T, c) & \stackrel{(3.3)}{=} \sum_{\substack{S \in \mathcal{L}(T), S^{*} \ni i}} \gamma(T, S)[c(S)-c(S \backslash i)] \\
& \stackrel{(3.4)}{=} \frac{1}{\left|T^{*}\right|}[c(T)-c(T \backslash i)]+\sum_{\substack{S \in L(T) \backslash T \\
S^{*} \ni i}} \frac{1}{\left|T^{*}\right|} \sum_{j \in T^{*} \backslash S} \gamma(T \backslash j, S)[c(S)-c(S \backslash i)] \\
= & \frac{1}{\left|T^{*}\right|}[c(T)-c(T \backslash i)]+\sum_{j \in T^{*} \backslash i} \frac{1}{\left|T^{*}\right|} \sum_{\substack{S \in L(T \backslash j) \\
S^{*} \ni i}} \gamma(T \backslash j, S)[c(S)-c(S \backslash i)] \\
& \stackrel{(3.3)}{=} \frac{1}{\left|T^{*}\right|}\left[c(T)-c(T \backslash i)+\sum_{j \in T^{*} \backslash i} \phi_{i}^{r}(T \backslash j, c)\right] .
\end{aligned}
$$

(b) $i \in T \backslash T^{*}$.

We have

$$
\begin{aligned}
\phi_{i}^{r}(T, c) & \stackrel{(3.3)}{=} \sum_{\substack{S \in \in(T), S^{*} \ni i}} \gamma(T, S)[c(S)-c(S \backslash i)] \\
& \stackrel{(3.4)}{=} \sum_{\substack{S \in \in(T), S^{*} \rightarrow i}} \frac{1}{\left|T^{*}\right|} \sum_{j \in T^{*} \backslash S} \gamma(T \backslash j, S)[c(S)-c(S \backslash i)] \\
& =\sum_{j \in T^{*}} \frac{1}{\left|T^{*}\right|} \sum_{\substack{S \in \mathcal{L}(\backslash \backslash j) \\
S^{*} \ni i}} \gamma(T \backslash j, S)[c(S)-c(S \backslash i)] \\
& \stackrel{(3.3)}{=} \frac{1}{\left|T^{*}\right|} \sum_{j \in T^{*}} \phi_{i}^{r}(T \backslash j, c) .
\end{aligned}
$$

We finish the proof by showing that for all $T \in \mathcal{L}(P), T \neq \emptyset, \quad \phi^{r}(T, c)$ is a cost allocation. By assumption (A), induction on the coalition size $|T|$ is allowed. If $T=\{i\}, \phi_{i}^{r}(T, c)=c(T)$. Suppose $|T|>1$. We can use the recursive form as 
follows:

$$
\begin{aligned}
\sum_{i \in T} \phi_{i}^{r}(T, c) & \stackrel{(3.5)}{=} \sum_{i \in T^{*}} \frac{1}{\left|T^{*}\right|}\left[c(T)-c(T \backslash i)+\sum_{j \in T^{*} \backslash i} \phi_{i}^{r}(T \backslash j, c)\right] \\
& +\sum_{i \in T \backslash T^{*}} \frac{1}{\left|T^{*}\right|} \sum_{j \in T^{*}} \phi_{i}^{r}(T \backslash j, c) \\
& =c(T)-\frac{1}{\left|T^{*}\right|} \sum_{i \in T^{*}} c(T \backslash i)+\sum_{i \in T} \frac{1}{\left|T^{*}\right|} \sum_{j \in T^{*} \backslash i} \phi_{i}^{r}(T \backslash j, c) \\
& =c(T)-\frac{1}{\left|T^{*}\right|} \sum_{i \in T^{*}} c(T \backslash i)+\sum_{j \in T^{*}} \frac{1}{\left|T^{*}\right|} \sum_{i \in T \backslash j} \phi_{i}^{r}(T \backslash j, c) \\
& =c(T)-\frac{1}{\left|T^{*}\right|} \sum_{i \in T^{*}} c(T \backslash i)+\frac{1}{\left|T^{*}\right|} \sum_{j \in T^{*}} c(T \backslash j) \\
& =c(T) .
\end{aligned}
$$

Note that in case $\mathcal{L}(T)=2^{T}$, where $T \in \mathcal{L}(P) \backslash \emptyset$, (3.5) changes into (3.2). Moreover, in case $\mathcal{L}(T)=\{S \subseteq T \mid$ if $j \in S$ then $i \in S$ for all $i \preceq j\}$, where $T \in \mathcal{L}(P) \backslash \emptyset$, the Recursive Shapley value does not coincide with the Shapley value under precedence constraints introduced by Faigle and Kern [1992].

Proposition 3.2 states that the Recursive Shapley value is a probabilistic marginalistic allocation rule in that the allocation to any player is some expected outcome of his marginal contributions in the game.

Proposition 3.2. $\phi^{r}$ is a probabilistic marginalistic allocation rule, that is, for all $T \in \mathcal{L}(P), S \in \mathcal{L}(T), S \neq \emptyset$, the coefficients $\gamma(T, S)$ are positive and for all $T \in \mathcal{L}(P), i \in T, \sum_{\substack{S \in \mathcal{L}(T), S^{*} \rightarrow i}} \gamma(T, S)=1$.

Proof: It is straightforward to see that the real numbers $\gamma(T, S)$ are positive for all $T \in \mathcal{L}(P), S \in \mathcal{L}(T), S \neq \emptyset$. To prove that for all $T \in \mathcal{L}(P), i \in T$,

$\sum_{\substack{S \in \mathcal{L}(T), S^{*} \rightarrow i}} \gamma(T, S)=1$, we use induction on the coalition size $|T|$ which is allowed by assumption (A). If $T=\{i\}$, then $\gamma(T, T)=1$. Suppose $T \in \mathcal{L}(P),|T|>1$ and $i \in T$. There are two cases.

(a) $i \in T^{*}$. 
We have

$$
\begin{aligned}
\sum_{\substack{S \in \mathcal{L}(T), S^{*} \ni i}} \gamma(T, S) & =\gamma(T, T)+\sum_{\substack{S \in \mathcal{L}(T) \backslash T, S^{*} \ni i}} \gamma(T, S) \\
& \stackrel{(3.4)}{=} \frac{1}{\left|T^{*}\right|}+\frac{1}{\left|T^{*}\right|} \sum_{\substack{S \in \mathcal{L}(T) \backslash T, S^{*} \ni i}} \sum_{j \in T^{*} \backslash S} \gamma(T \backslash j, S) \\
& =\frac{1}{\left|T^{*}\right|}+\sum_{j \in T^{*} \backslash i} \frac{1}{\left|T^{*}\right|} \sum_{\substack{S \in \mathcal{L} \\
S^{*} \ni i}} \gamma(T \backslash j, S) \\
& =\frac{1}{\left|T^{*}\right|}+\sum_{j \in T^{*} \backslash i} \frac{1}{\left|T^{*}\right|} \quad \text { (by the induction hypothesis) } \\
& =1 .
\end{aligned}
$$

(b) $i \in T \backslash T^{*}$.

We have

$$
\begin{aligned}
& \sum_{\substack{S \in \mathcal{L}(T), S^{*} \ni i}} \gamma(T, S) \stackrel{(3.4)}{=} \frac{1}{\left|T^{*}\right|} \sum_{\substack{S \in \mathcal{L}(T), S^{*} \ni i}} \sum_{j \in T^{*} \backslash S} \gamma(T \backslash j, S) \\
& =\quad \sum_{j \in T^{*}} \frac{1}{\left|T^{*}\right|} \sum_{\substack{S \in \mathcal{L}(T \backslash j) \\
S^{*} \ni i}} \gamma(T \backslash j, S) \\
& =\sum_{j \in T^{*}} \frac{1}{\left|T^{*}\right|} \quad \text { (by the induction hypothesis) } \\
& =1 \text {. }
\end{aligned}
$$

As mentioned in the introduction, a cost allocation should be "fair" in some sense. A possible way to define fair allocations is to demand that they are in the core of a cooperative game. The idea of the core of a game essentially goes back to von Neumann and Morgenstern [1944]. $\operatorname{core}(N, c)$ is the set of all allocations $x \in \mathbb{R}^{N}$ for which there is no coalition $S \subseteq N$ such that $x(S)>c(S)$, which means that no coalition should have to pay more than its cost. It turns out that the Shapley value lies not in the core for a general cooperative game. However, for the class of the so-called quasi-convex games, Sprumont [1990] proved that the Shapley value belongs to the core.

In our generalized model, for a given subfamily $\mathcal{L}(P)$, we define $\operatorname{core}(P, c)=\left\{x \in \mathbb{R}^{N} \mid x(N)=c(N)\right.$ and $x(S) \leq c(S)$ for all $\left.S \in \mathcal{L}(P) \backslash N\right\}$. 
We make one extra assumption to hold for a subfamily $\mathcal{L}(P)$. It is straightforward to see that, besides $\mathcal{L}(P)=2^{N}$, (1.2) and (1.3) also satisfy this assumption.

(B) $S \cap T^{*} \subseteq S^{*}$ for all $T \in \mathcal{L}(P), S \in \mathcal{L}(T)$.

A cooperative game $(P, c)$ is called quasi-concave if

$$
\sum_{i \in S \cap T^{*}}[c(S)-c(S \backslash i)] \geq \sum_{i \in S \cap T^{*}}[c(T)-c(T \backslash i)] \text { for all } T \in \mathcal{L}(P), S \in \mathcal{L}(T) .
$$

Clearly, this definition corresponds with the original definition of quasi-convexity as given by Sprumont [1990] if $\mathcal{L}(P)=2^{N}$. Note that, because of assumption (B), $S \backslash i \in \mathcal{L}(P)$ for all $T \in \mathcal{L}(P), S \in \mathcal{L}(T)$ and $i \in S \cap T^{*}$.

In the following theorem, we generalize the result of Sprumont [1990] for subfamilies $\mathcal{L}(P)$ not necessarily equal to $2^{N}$. The recursive formula of $\phi^{r}$ turns out to be very useful in the proof.

Theorem 3.2. Let $\mathcal{L}(P)$ be a subfamily that satisfies assumption $(\mathbf{A})$ and $(\mathbf{B})$. If the cooperative game $(P, c)$ is quasi-concave, then $\phi^{r}(P, c) \in \operatorname{core}(P, c)$.

Proof: If $(P, c)$ is quasi-concave, then each subgame $(S, c)$ is quasi-concave for all $S \in \mathcal{L}(P)$. Furthermore, by assumption (A), we can use induction on $|N|$. If $N=\{i\}, \quad \phi_{i}^{r}(P, c)=c(\{i\})$. Suppose $n \geq 2$ and $T \in \mathcal{L}(P)$.

$$
\begin{aligned}
\sum_{i \in T} \phi_{i}^{r}(P, c) & \stackrel{(3.5)}{=} \sum_{i \in N^{*} \cap T} \frac{1}{\left|N^{*}\right|}[c(N)-c(N \backslash i)]+\sum_{i \in T} \frac{1}{\left|N^{*}\right|} \sum_{j \in N^{*} \backslash i} \phi_{i}^{r}(N \backslash j, c) \\
& =\frac{1}{\left|N^{*}\right|} \sum_{i \in N^{*} \cap T}[c(N)-c(N \backslash i)]+\frac{1}{\left|N^{*}\right|} \sum_{j \in N^{*} \cap T} \sum_{i \in T \backslash j} \phi_{i}^{r}(N \backslash j, c) \\
& +\frac{1}{\left|N^{*}\right|} \sum_{j \in N^{*} \backslash T} \sum_{i \in T} \phi_{i}^{r}(N \backslash j, c) \\
& \leq \frac{1}{\left|N^{*}\right|} \sum_{i \in N^{*} \cap T}[c(N)-c(N \backslash i)]+\frac{1}{\left|N^{*}\right|} \sum_{j \in N^{*} \cap T} c(T \backslash j) \\
& +\frac{1}{\left|N^{*}\right|} \sum_{j \in N^{*} \backslash T} c(T) \quad(\text { by }(\mathrm{B}) \text { and the induction hypothesis }) \\
& \leq \frac{1}{\left|N^{*}\right|} \sum_{i \in N^{*} \cap T}[c(T)-c(T \backslash i)]+\frac{1}{\left|N^{*}\right|} \sum_{j \in N^{*} \cap T} c(T \backslash j)
\end{aligned}
$$




$$
\begin{aligned}
& +\frac{1}{\left|N^{*}\right|} \sum_{j \in N^{*} \backslash T} c(T) \quad \text { (by quasi-concavity of }(P, c) \text { ) } \\
& =\frac{1}{\left|N^{*}\right|}\left[\left|N^{*} \cap T\right|+\left|N^{*} \backslash T\right|\right] c(T) \\
& =c(T) .
\end{aligned}
$$

In Paulusma [1997], more information on the two extensions of the Shapley value can be found. For which poset structures, there exist efficient algorithms for the deterministic evaluation of these values is still an open problem.

\section{REFERENCES}

[1] J.M. Bilbao and P.H. Edelman [1998]: Axioms for the Shapley value on convex geometries. European Journal of Operational Research 110, 368-376.

[2] T.S.H. Driessen [1991]: A survey of consistency properties in cooperative game theory. SIAM Review 33, 43-59.

[3] R.A. Evans [1996]: Value, consistency, and random coalition formation. Games and Economic Behavior 12, 68-80.

[4] U. Faigle and W. Kern [1992]: The Shapley value for cooperative games under precedence constraints. Int. Journal of Game Theory 21, 249-266.

[5] U. Faigle and W. Kern [1997]: On the core of submodular cost games. Preprint, University of Twente. To appear in: Mathematical Programming.

[6] J. von Neumann and O. Morgenstern [1944]: Theory of Games and Economic Behavior. Princeton University Press, Princeton, U.S.A.

[7] D. Paulusma [1997]: Extensions of the Shapley Value for TU-Games defined on Posets. Master's thesis, Faculty of Mathematical Sciences, University of Twente, Enschede, The Netherlands.

[8] A.E. Roth (editor) [1988]: The Shapley Value: Essays in honor of Lloyd S. Shapley. Cambridge University Press, Cambridge, U.S.A.

[9] L.S. Shapley [1953]: A Value for n-Person Games. Annals of Mathematics Study 28, 307-317 (Princeton University Press). Also in [8], 31-40.

[10] Y. Sprumont [1990]: Population monotonic allocation schemes for cooperative games with transferable utility. Games and Economic Behavior 2, 378-394.

[11] R.J. Weber [1988]: Probabilistic values for games. In [8], 101-119.

Faculty of Mathematical Sciences, University of Twente, P.O.Box 217, 7500 AE ENSCHEDE, THE NETHERLANDS

E-mail address: $\{$ driessen, paulusma\}@math.utwente.nl 